\title{
REEB STABILITY FOR NONCOMPACT LEAVES IN FOLIATED 3-MANIFOLDS
}

\author{
JOHN CANTWELL ${ }^{1}$ AND LAWRENCE CONLON ${ }^{2}$
}

\begin{abstract}
Conditions are investigated under which a proper leaf has a trivally foliated, saturated neighborhood.
\end{abstract}

We consider transversely orientable foliations of closed, orientable 3-manifolds by (necessarily orientable) surfaces. These hypotheses will be assumed throughout, save mention to the contrary.

Definition. A surface $L$ has the $C^{r}$-stability property if, whenever $L$ is homeomorphic to a proper leaf of a $C^{r}$ foliation $\mathscr{F}$, that leaf admits an open, $\mathscr{F}$-saturated neighborhood $U$, fibered over $S^{1}$ by $\mathcal{F} \mid U$.

Definition. A surface $L$ has the weak $C^{r}$-stability property if, whenever $L$ is homeomorphic to a proper leaf of a $C^{r}$ foliation $\mathscr{F}$, such that the germinal holonomy group of the leaf is trivial, then that leaf admits an open, $\mathscr{F}$-saturated neighborhood $U$, fibered over $\mathbf{R}$ by $\mathcal{F} \mid U$.

In [In], it was shown (essentially) that the plane has the $C^{1}$-stability property and it was announced that every surface with finitely generated fundamental group has the weak $C^{1}$-stability property. This condition on the fundamental group implies that the surface has only finitely many ends and finitely many handles (for the concept of "ends", see [A-S], [Ri], [C-C1], etc.).

For $C^{2}$ foliations, these results generalize considerably.

THEOREM 1. Every orientable surface with at most countably many ends and finitely many handles has the weak $C^{2}$-stability property.

THEOREM 2. Every orientable surface with at most countably many ends and no handles has the $C^{2}$-stability property.

ReMarks. (1) By the constructive proof of [C-C1, Theorem 2], the condition in Theorem 2 does not imply the $C^{1}$-stability property. In fact, the cylinder is not $C^{1}$-stable.

(2) It is easy to construct an example showing that the surface with two ends, both nonplanar (hence with infinitely many handles), does not have the weak $C^{\infty}$-stability property [Im, p. 622].

Received by the editors October 2, 1980.

1980 Mathematics Subject Classification. Primary 57R30.

${ }^{1}$ Partially supported by NSF Grant MCS-8001547.

${ }^{2}$ Partially supported by NSF Grant MCS-8003248. 
(3) As was shown in [C-C1, (6.2)] an impressively large array of surfaces satisfying the conditions of our theorems actually occur as proper leaves in suitable $C^{\infty}$ foliations of all closed, orientable 3-manifolds.

(4) The countability condition on the endset is probably essential in both theorems. We have an example in which the surface with a Cantor set of ends and no handles appears as a nonstable, proper leaf with trivial germinal holonomy, but it is not clear that the construction can be $C^{2}$-smoothed.

(5) While the notion of "handle" (a closed, nonseparating submanifold of codimension one) is defined in higher dimensions, it is not possible to generalize Theorem 2 to higher dimensions. Examples constructed in [C-C4, \$4] give, for each integer $n \geqslant 3, n$-dimensional manifolds with one end and no handles, appearing as proper leaves with nontrivial germinal holonomy in suitable closed, $C^{\infty}$-foliated $(n+1)$-manifolds.

(6) In higher dimensions (but not in higher codimensions) it seems reasonable to conjecture that, whenever $\pi_{1}(L)$ is finitely generated and $H^{1}(L ; \mathbf{R})=0$, then $L$ will have the $C^{2}$-stability property. Indeed, a theorem claimed by P. Dippolito [Di1, Theorem 4] and subsequently disclaimed by him [Di2], would imply, via the result of W. Thurston [Th], [R-S], [Sch], that such a leaf is $C^{1}$-stable. Dippolito's theorem claims weak $C^{0+}$-stability for manifolds with finitely generated fundamental group, and we know of no counterexample. ${ }^{3}$

Proofs of THE THEOREMS. By [C-C3, (4.0)], a proper leaf $L$ is necessarily at finite level and has closure a finite union of local minimal sets $X_{i}$. Clearly no $X_{i}$ can be of locally dense type. By [Du], if some $X_{i}$ is of exceptional type, the endset $\mathcal{E}(L)$ will contain a copy of the Cantor set. Thus, the countability of $\mathscr{E}(L)$ implies that each $X_{i}$ is a proper leaf. By [C-C3, (6.0)], such a leaf $L$ has polynomial growth and finite type (also, cf. [Ts]).

Since $L$ is a proper leaf with nonexponential growth, the amputation process of [C-C2, \$7] can be applied. This changes the ambient 3-manifold $M$ and the foliation $\mathscr{F}$, and it replaces $L$ by the surface obtained by adjoining to $L$ all of its isolated planar ends. Remark that all ends of $L$ are planar under our hypotheses; hence the fact that $L$ is of finite type enables us to iterate the amputation process a finite number of times so as to produce a new $C^{2}$-foliated 3-manifold $M^{*}$ in which the original leaf $L$ has been transformed into a compact leaf $L^{*}$ of the new foliation $\mathcal{F}^{*}$.

In the hypotheses of Theorem 1 , the compact leaf $L^{*}$ has trivial germinal holonomy; hence standard Reeb stability implies that $L^{*}$ has an $\mathscr{F}^{*}$-saturated neighborhood $U^{*}$, fibered over $\mathbf{R}$ by $\mathscr{F} * \mid U^{*}$.

In the hypotheses of Theorem $2, L^{*}$ will be homeomorphic to $S^{2}$ and standard Reeb stability implies that $\mathcal{F}^{*}$ fibers $M^{*}$ over $S^{\mathbf{l}}$.

In both cases, the sequence of amputations is reversed by a finite sequence of foliated surgeries. In each surgery, one first inserts Reeb components along finitely many closed transversals in the standard way. Then the interiors of some of these

${ }^{3} \mathrm{~T}$. Inaba has recently constructed a counterexample to Dippolito's theorem in dimension five. 
Reeb components are deleted and some of the resulting toral faces are glued together pairwise. To the remaining toral faces are glued suitably foliated 3-manifolds having toral boundary leaves. It is easy to follow a finite iteration of such processes back to the original foliated manifold so as to verify the conclusions of the respective theorems.

\section{REFERENCES}

[A-S] L. V. Ahlfors and L. Sario, Riemann surfaces, Princeton Univ. Press, Princeton, N. J., 1960, Chapter I, 86.

[C-C1] J. Cantwell and L. Conlon, Leaf prescriptions for closed 3-manifolds, Trans. Amer. Math. Soc. 236 (1978), 239-261.

[C-C2] _ Leaves with isolated ends in foliated 3-manifolds, Topology 16 (1977), 311-322.

[C-C3] _ Poincaré-Bendixson theory for leaves of codimension one, Trans. Amer. Math. Soc. 265 (1981), 181-209.

[C-C4] __ Tischler fibrations of open, foliated sets, Ann. Inst. Fourier (Grenoble) (to appear).

[Di1] P. Dippolito, Codimension one foliations of closed manifolds, Ann. of Math. 107 (1978), 403-453.

[di2] __ Corrections to codimension one foliations of closed manifolds, Ann. of Math. 110 (1979), 203.

[Du] G. Duminy (to appear).

[Im] H. Imanishi, On the theorem of Denjoy-Sacksteder for codimension one foliations without holonomy, J. Math. Kyoto Univ. 14 (1974), 607-634.

[In] T. Inaba, On stability of proper leaves of codimension one foliations, J. Math. Soc. Japan 29 (1977), 771-778.

[Ri] I. Richards, On the classification of noncompact surfaces, Trans. Amer. Math. Soc. 106 (1963), 259-269.

[R-S] G. Reeb and P. Schweitzer, Un théorème de Thruston établi au moyen de l'analyse non standard, Lecture Notes in Math., Vol. 652, Springer-Verlag, New York, 1978, p. 138.

[Sch] W. Schachermayer, Une modification standard de la demonstration non standard de Reeb et Schweitzer, Lecture Notes in Math., Vol. 652, Springer-Verlag, New York, 1978, pp. 139-140.

[Th] W. Thurston, A generalization of the Reeb stability theorem, Topology 13 (1974), 347-352.

[Ts] N. Tsuchiya, Leaves of finite depth (to appear).

Department of Mathematics, St. Louis University, St. Louis, Missouri 63103

Department of Mathematics, Washington University, St. Louis, Missouri 63103 